\title{
Assessing the Impacts of "Infodemic" on Social Well-Being and Health Preparedness of Indian Communities during COVID-19 Pandemic through WhatsApp groups study
}

\author{
Syeda Saadia Azim ${ }^{1}$, Dipayan Dey $^{2}$ and Arindam Roy ${ }^{2 *}$ \\ ${ }^{1}$ The American Center, U. S. Consulate, Kolkata, India \\ ${ }^{2}$ South Asian Forum for Environment, Kolkata, India
}

\begin{abstract}
The present study is an attempt to understand the propagation of health-related fake news in India during COVID-19 pandemic in diversely distributed WhatsApp groups comprising uneven groups of members. Ten different WhatsApp groups were selected covering demographic and socio-economic profile and ten most important health-related fake news were studied. Different fake news circulation paths like forward, react, multi-forward, reference etc. were analyzed for different groups. It was observed that older generations (boomers) were more susceptible towards fake news and they tended to distribute the fake news in different groups. Male boomers actively circulate pseudo-science information whereas female boomers distribute faith-based information in the WhatsApp group. It was found that the Gen X as well as Millennials played a role as the first recipients or first responders but primarily most of the forwards were attributed to the Boomers of both genders. Levels of education, social media influence or ignorance, technology access, age, gender and understanding played a major role in the distribution and propagation of fake information during times of COVID. It can be also inferred that easy availability of data and devices, lack of understanding of the game of social media and unscientific baggage of age old beliefs were some factors for over -dependence and reliance on fake news.
\end{abstract}

KEYWORDS: Fake News, WhatsApp Group, Misinformation, COVID-19

*arindam.safe@gmail.com 


\subsection{Introduction}

Five weeks prior to declaring COVID-19 a pandemic, WHO described the bigger challenge of handling the enormous volume of information that people were exposed globally affecting the sanity of human minds in times of pandemic (Azim et al. 2020). The director of the organization pointed out the limits of human minds that filters real over fake and the inability to debunk falsity from actual as a public health issue. Increasing fake news in the social media on the new internet era highlights the erosion of long-standing institutional bulwarks against misinformation in the society. It is a global concern and systemic safeguard is needed to stop fake and malicious information flow (Lazer et al 2018). Studies reveal that analytic thinking correlates negatively with perceived accuracy of fake news and positively with media truth discernment whereas it is not moderated by the presence or absence of information or by prior familiarity with the news (Berduygina et al, 2019). It suggests that belief in fake news has similar cognitive properties to other forms of falsified receptivity and reinforces the important role of analytic thinking in recognizing misinformation (Pennycook and Rand 2018).

The massive societal impact of false news and misinformation on community perception and attitude has been asserted earlier (Brewer et al 2013; Gupta et al 2014; Allcott \& Matthew 2017). Post COVID-19, the global pandemic of 'Fake News', defined today as the amalgamation of information, misinformation, dis-information, mal-information that forms a malicious mix of information posing greater threat to public wellbeing, is now been christened as the "Infodemic" and has been identified as a major cause of global unrest and worry (Bharali and Goswami, 2017, Bali and Desai, 2019). Infodemic (pronounced as // infə' ${ }^{\prime} \mathrm{demik} / / \mathrm{Inf} \mathrm{I}^{\prime} \mathrm{d} \varepsilon \mathrm{mIk} /$ ) is an early twenty first century word originated from a blend of pandemic and epidemic. In the lexicon dictionary it means "a surfeit of information about a problem that is viewed as being a detriment to its solution". (https://www.lexico.com/en/definition/infodemic). World leaders are addressing the issue of an "Infodemic" by prioritising its solution to safeguard sanity of the world. Since such "Infodemic" can lead to poor decisions about consequential matters and is persistent and difficult to correct, debunking it is an important scientific and public-policy goal (Sally et al 2017), even quashing rumours through direct refutation may even facilitate its deeper penetration (Berinsky 2015). In the era of equitable internet access, filtering, vetting, and verifying online information continues to be essential in library and information science, as the lines between traditional news and online information are blurring (Rubin et al 2015).

Information mines are developed to empower people but have otherwise endangered the wisdom and well-being at the same time. In India with its 1.3 billion people, severely diverse, and socially classified is very adversely affected due to the infodemic. The segregation of news and how people pose their trust on it is a great concern of policy makers. With several instances of social disruptions and severe public health risks, the looming infodemic poses a challenge not less than the pandemic itself. With over 560 million Internet users in India, it is the second largest market of thernet world 
(https://www.statista.com/topics/2157/internet-usage-in-india/). In combating the pandemic, since the world has gone virtual there has been an exponential growth of unverified data content and information that has little control of the knowledgeable or subject matter experts. The large volume of the misleading, false or partially correct information flow has impacted social well-being of people. This infodemic, created with deceitful purposes of political leverage, vested interest or even by innocence has revoked potential social and health risks. Reviewing literature suggests that the knowledge about the dynamics of the life cycle of a social media rumor is scarce and robust machine learning techniques that can provide assistance in real time for assessing the veracity of rumors is highly needed (Zubiaga et al 2016). The present paper intends to assess the socio-psychological impact of "Infodemic" during the pandemic that created health impacts on people in the Indian subcontinent.

\subsection{Methodology}

The impact of false news over Indians during the pandemic was studied by randomly selecting 10 families and professional WhatsApp groups with minimum twenty people or more as members, using feedback surveys and attitude scaling (Lickert et al 2007). Focused group discussions (FGDs) were made amongst cross-sectoral socio-economic groups and as well age ranges. Gender perception was studied statistically to understand the gender bias, if any.

100 unique mis-dis-mal information instances were also sampled from these WhatsApp groups and a matrix was prepared to assess how they were shared on popular online platforms categorizing them for COVID related public health information. Systematically each story was explained for thematic categories, sources and origins of claims, and their trust factor leading to responses from the receivers of the information. Out of these ten most important stories based on the risk factor they generated in public health are discussed herein, for reference (Table 1). Altogether, 1398 participants were interviewed. Each of the group members were separately interviewed and altogether 1398 participants were asked questions about their activity in WhatsApp.

Table 1: Selected fake news for the present study

\begin{tabular}{lllll}
\hline SN & Fake News & Type & Time & Ref \\
\hline 1. & Hydroxy-chloroquine can cure COVID & Dis-info & 6 weeks & 1 \\
2. & Garlic and hot water are effective protector & Mis-info & 1 week & 2 \\
3. & Drinking cow urine can cure COVID & Dis-info & 2 weeks & 3 \\
4. & Pneumonia vaccines/antibiotics help mitigate COVID & Dis-info & 2 weeks & 4 \\
5. & Indians have stronger immunity & Mal-info & 4 weeks & 5 \\
6. & $\begin{array}{l}\text { Anti-tuberculosis BCG-vaccine protected people against } \\
\text { corona virus }\end{array}$ & Dis-info & 2 weeks & 6
\end{tabular}




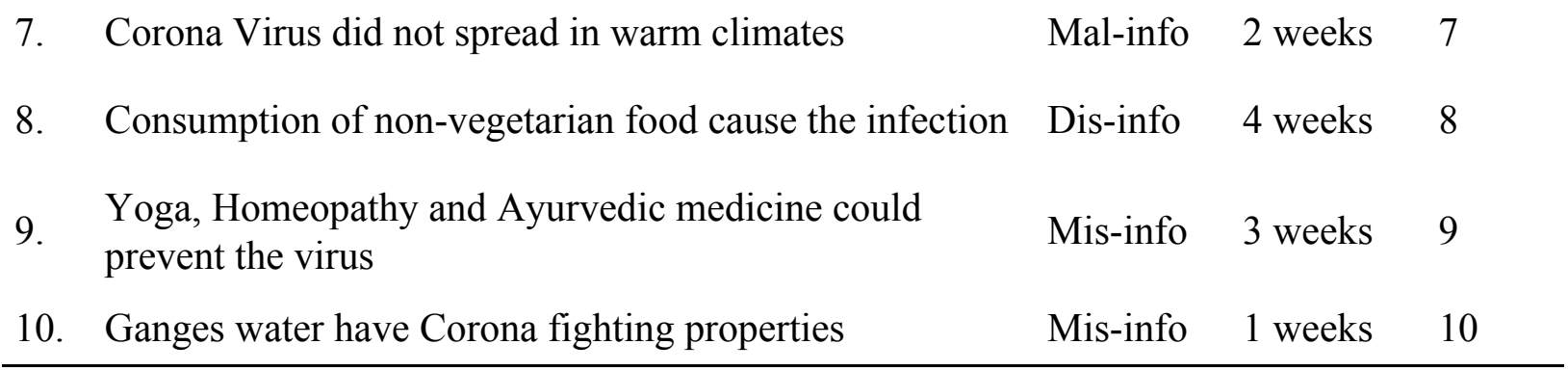

${ }^{1}$ Boomslive.in, ${ }^{2,4,6,8,9}$ Generic, ${ }^{3,10}$ IndiaTimes, ${ }^{5}$ Livemint, ${ }^{7}$ News 18

\subsection{Results}

The study of responding and non-responding participants brought forth some interesting facts about how people reacted to the above series of misinformation, dis-information and Malinformation in WhatsApp group.

\subsection{Group-wise different WhatsApp activity}

Different WhatsApp activities have been classified for analysis purpose; i.e. Re-forwards (forwarded messages from elsewhere), Reactions (such as great, emoticons, thank you etc.), Many Forwards (accepted to forward in another group), No Response (Silent participants), References (refer to the WhatsApp message while talking), Adverse Reaction (questioning the message). The normalized activities for each group have been represented as heat-map (Fig 1). Separately and allover, adverse reaction was found to be the least performed activity comprising $0-12 \%$ of all the activity. The observation indicates the counter-question against fake news is minimal among the participants. On the other hand, with a median of $32.4 \%$, silent participants are dominating the proportion of all groups. Among the other reactions forward, references and reactions hold a substantial proportion of group participants.

These groups can be classified as school groups (Group C, G, I), community groups with diverse demographic profiles (Group D, E, H, J), professional group (Group F), Family Group (Group B) and acquaintance group (Group A).

The pattern of different activities among the school groups is very similar as observed from the correlation analysis (Correlation coefficient: $0.81-0.96$, p-value $<0.1$ ). The different community groups also significantly correlated with their activities $(0.62-0.97$, p-value $<0.1)$. Inactive members were predominant in community groups whereas among professional groups most number of active members were found. 


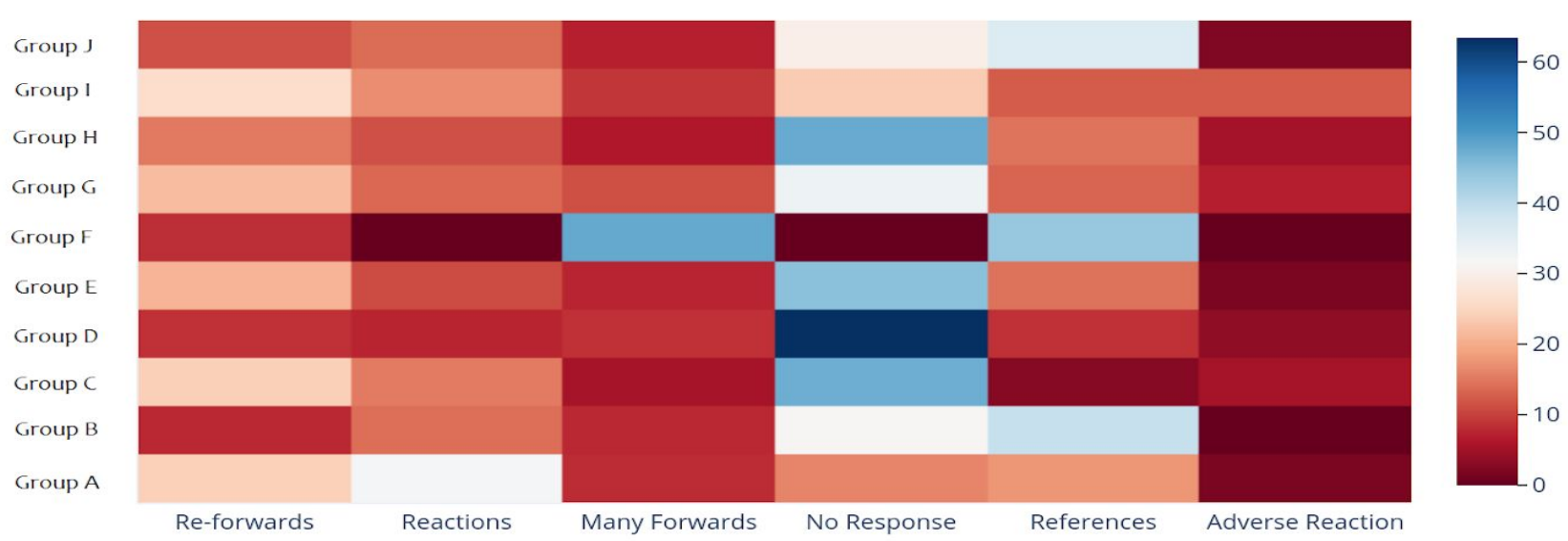

Fig1: Group-wise reactions on fake news (in percentile).

\subsection{Effect of age-group in fake news propagation}

The participants were grouped into four according to their age; i.e. Generation $Z(<22$ years), Millennials (22 - 38 years), Generation X (39 - 54 years), Boomers (55 - 73 years). The result clearly indicates the older generation (Boomers) directly forward/react/multi-forward/referred the fake news promptly compared to any other groups. More than 50\% (53.2\%) of the fake news circulation activity is done through the boomers followed by Generation X (22.3\%), Millennials (12.8\%) and Generation Z (11.7\%). It can be clearly inferring that the younger generation does not participate much in circulating fake news as compared to the older generation.

The one-to-one interview with the group member reveals $32 \%$ receivers of the fake messages forwarded it to their newer contacts. 54\% either used it effectively in times of daily conversation as a way to share the newly acquired knowledge. Rest, who did not show reactions online still did not debunk the information and remained passive spreaders. 27\% among the receivers though did not provide online responses attributed the piece of information during direct discussions with their other counterparts. All of them agreed that they weren't bothered about the authenticity of the information nor its origin. $75 \%$ of those, who were tracked, believed that the information had aspects of truth so they relied on them and had lasting impressions. $78 \%$ respondents agreed that they received the information from reliable sources such as friends and family so they believed in them. $35 \%$ respondents said they did not rely completely but since everyone else believed the information, they thought it could be true. Seventy percent of respondents did not feel there was anything unethical in it. 84\% respondents said that such information generated extreme feelings such as anger, hate, fear, uncertainty, de-motivation or even at times confidence, happiness, hope in them. 


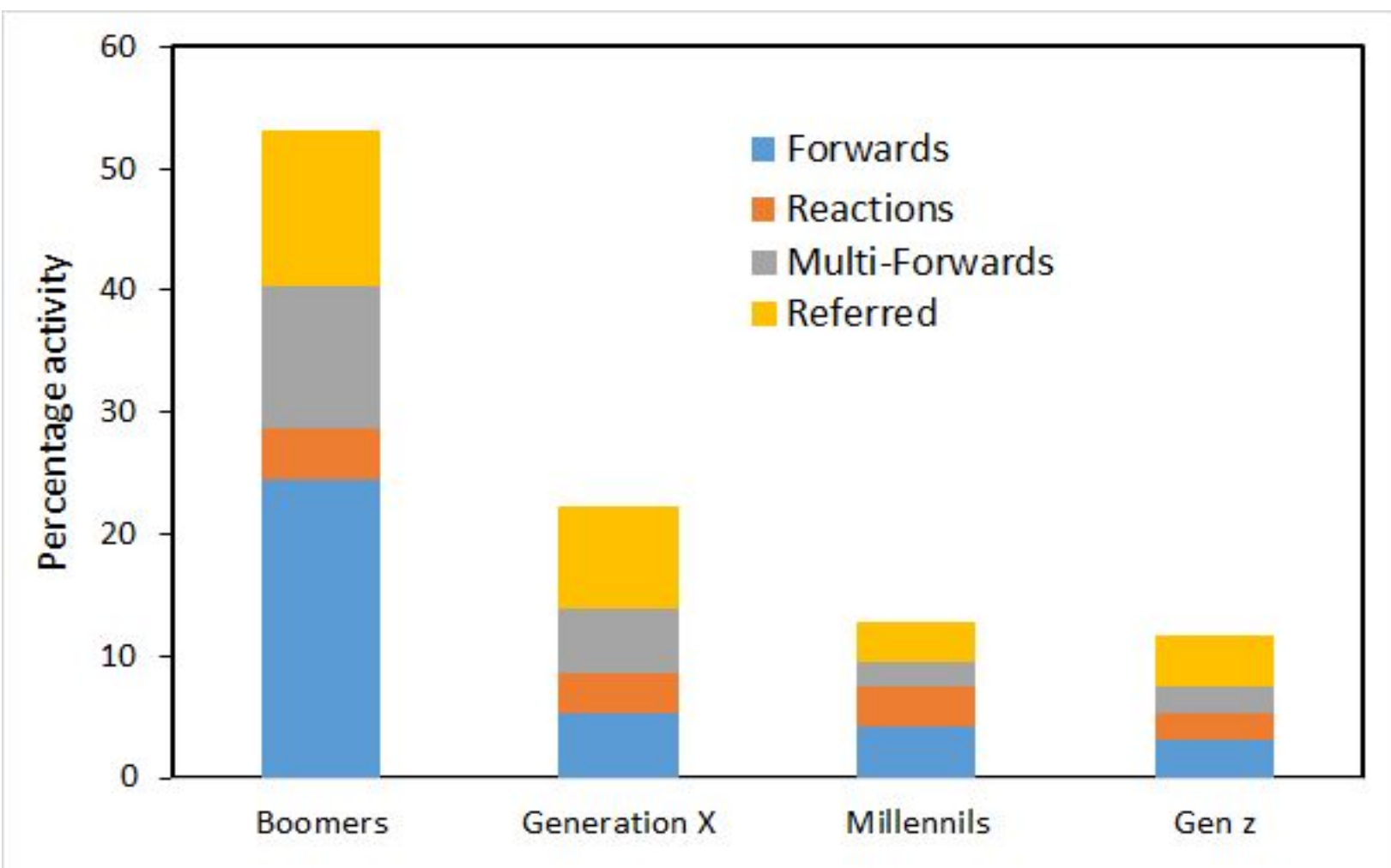

Fig 2: Percentage activity of different age group

\subsection{Effect of Gender in fake news propagation}

To evaluate the effect of gender on fake news propagation, the 10 selected fake news has been classified into two broad categories; faith-based news (SN - 2, 3, 8, 9, 10) and pseudoscience/mis-science based news ( $\mathrm{SN}-1,4,5,6,7)$. The result for male/female millennials and boomers has been represented in Fig 3. The faith-based information is majorly shared by females and the pseudo-science information is predominantly circulated through male population of the group. The trend has been observed for both millennials and the boomer population. Although the elderly female population shared more faith-based fake news compared to younger females. Similar trend was found in male population where elderly male has shared more pseudoscience based information compared to younger male. 


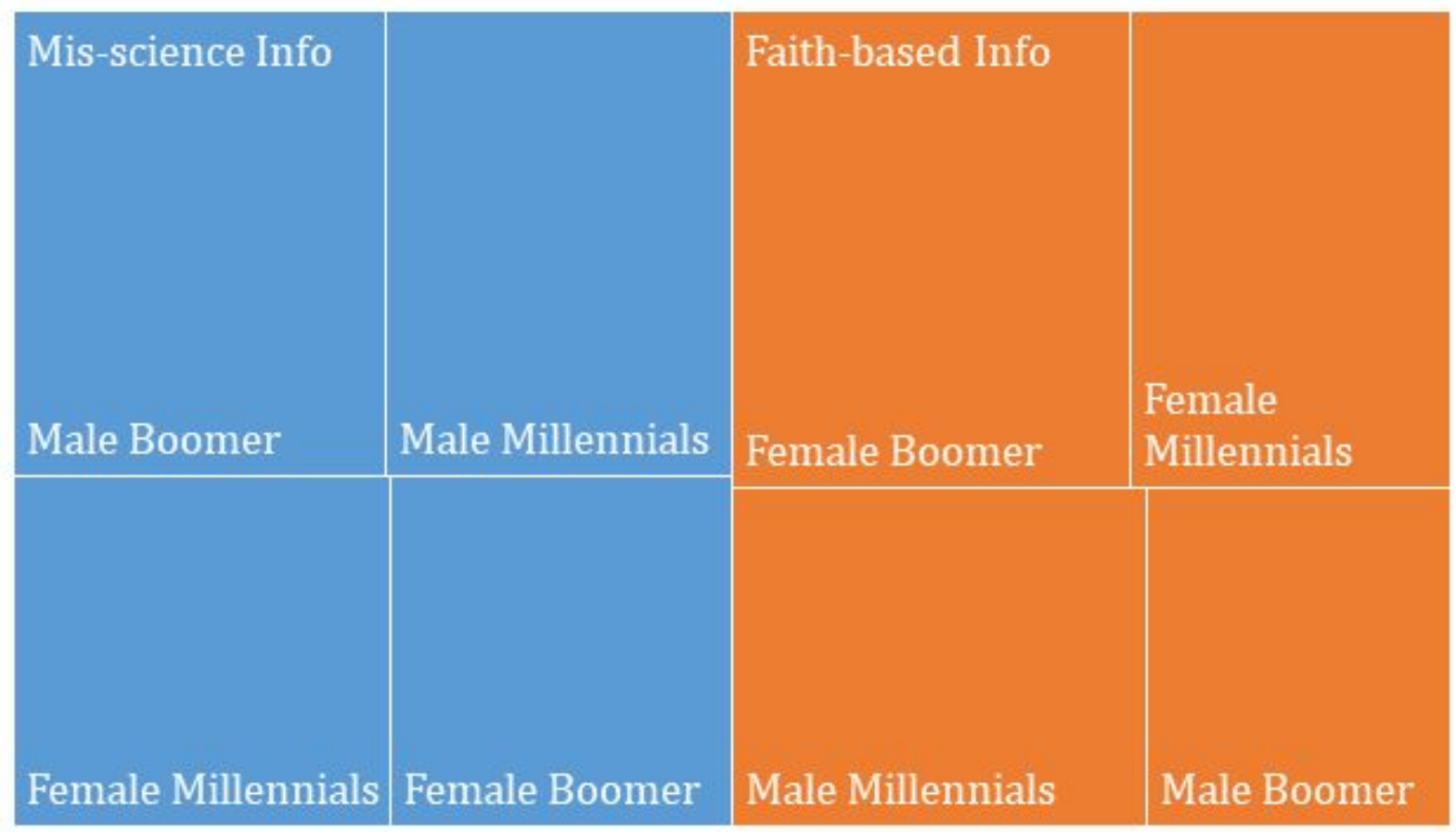

Fig 3: Gender-wise fake information circulation in WhatsApp group

\subsection{Results from Focus Group Discussion}

The online focus group discussion in small groups reveals several interesting facts. The oldest generation were the most vulnerable, they were the easy prey. But none were apologetic or convinced about the vulnerability and the threat they posed with the messages. No one apologized except two in the entire period of the study. This trait was attributed to their limited exposure to technology and contemporary social media tools. However, the fact that most participants have lived with pre-conceived half-truths all their lives were reasons enough to forward as endorsements to their belief. Most participants of that generation tend to depend on passover contents to remain relevant and active on social media. There were generational echo chambers that made most of the social media communities even in family, friends and social circles. Similar kinds of fake news circulated in the echo chambers and kept making rounds in family circles, social circles and community circles. They amplified the worst of pre-existent biases and unquestionable information that existed from pre COVID period.

\subsubsection{Trustworthiness of the sources}

People with low income and low educational level relied more on messages shared by their own than mainstream channels and newspapers. Their dependence on social media was much higher than mainstream media platforms. There was a big class divide in India between the middle income group and low income group who did not have access to TV. Most low income people yet depended on social media posts more than real time TV for their information. Though people accepted to rely more on scientists and doctors, and most fake news were in fact 
attributed to doctors and scientists without their knowledge rather than those who were passing on the information. But it was assessed that the reason was to create a false trust factor by naming the trustworthy and authentic sources rather than the piece of information. In several cases the scientists themselves had to debunk the false information circulating in their name.

\subsubsection{Factors dependent upon circulation of fake news}

In most cases people had not even watched or read the full piece of information before passing it on. The reason was less time, little empathy and little personal interest. Many times since the introduction matched with preconceived notions or key words in the person's mind motivated people to spread the word. In some of the cases it was revealed that innocent people who think they are helping friends and family by sharing what they think is legitimate medical advice.

\subsection{Discussion}

The brief research here in this portrays that no one knew the messenger and didn't bother about digging more on the message. The Indian Scientists Collective COVID-19 suggested that usually all fakes begin with portions of true content so its influence is never totally discredited. Indeed, much before the COVID -19 pandemic that held the world to standstill, Indians were already exposed to large amounts of false, purported, biased, innocent, faith-based, unscientific information to rule their lives. The spurt of social media in the last decade amplified its impact beyond imagination. There have been evidences of war conspiracies, terrorist attacks, communal tensions, health disasters, accidents, stampedes, violence, gender-based crimes, human trafficking etc. through the rapid spread of fake news in the country of 1.3 billion people. Although there are strict cyber and criminal laws that forbid creating, sharing, forwarding and editing of real information that can have adverse impact on social harmony, most Indians have had little understanding towards the "infodemic" thus bringing little regard for law and for being misguided by the anonymous. The reasons for such behaviors have been attributed to simpler traits; such as sheer entitlement, titillating joy, little knowledge, inherent biases, societal inequality, innocent behavior or little means to verify. The final falls out of fake and unreal content creations have been all the more dangerous, social evils such as lynching of human beings, acid attacks, domestic violence, rapes, extortions, suicides have been mainstreamed and overlooked by the larger community. Particularly during times of COVID-19, several remedial measures did the rounds based on hearsay, old beliefs, dependence on quacks and mistrust in governments posing greater threat towards the spread and effect of the disease. Although besides the health news, socio and economic effect has also been particularly devastating at times. There were instances of migrant laborers fleeing cities, covering thousands of miles barefooted, hungry, sick due to several bouts of misinformation and half-baked news. Instances of community clashes, social lockdowns and disruptions around took place due to news about purported spread of the virus, ultimately limiting the scope for alarm, preparedness and response towards the deadly disease. 
The present study clearly suggests that both gender and age played a major part in fake news propagation. Older generation are more likely to fall in the trap of fake news. The one-to-one conversation and Focus Group Discussion suggests that the older population often sends it out without reading or deeply thinking about. Limited exposure to social media tools and blind faith on anything published create an inhibition against checking the credibility. The trend of remaining active and creating a self-identity in social media are becoming a new edge thing for the boomer generation. It was observed that in the groups with diverse socio-economic profiles and low degrees of closeness tends to propagate fake news less likely whereas groups belonging to close members and lower socio-economic variability are more vulnerable in redistributing fake news. Females are more vulnerable towards faith-based doctored information whereas male are more likely to be manipulated by pseudo-science based fake news.

\section{CONCLUSION}

Indeed, every outbreak of disease or societal disorder is accompanied by a kind of tsunami of information that intends to safeguard one's interest at the cost of others. That information is backed by vested interest, business necessity, business rivalry, misinformation, rumors, etc since the middle ages. (https://www.elon.edu/u/imagining/surveys/viii-2017/future-of-the-information-environment/ ). The impact now is a million times more with social media amplifying and going faster and further, like the viruses that travel with people faster and further. This is the new challenge caused by the travel time of information and needs to be fixed by debunking the fake portion of the information with correct content. The correct version has to travel faster to be able to mitigate the impact. The challenge is not only to make sure that people are informed; it is also about making sure if people are informed to act appropriately, much ahead of fake news reaching them.

The reactions and comments on the information shared showed the growing sense of false trust and a sense of bloated confidence among respondents. Not a single discussion raised distrust or questioned the intention, wisdom or source of information on that content. It was found that people posed more suspicion on mainstream media and accused many of being biased citing individual, social and political ideologies. It appeared to do more harm than good to the public information consumption system even at the cost of public health risks. More than fifty percent of people in casual private chats passed on the information as their own and encouraged others to spread the unverified content. The fact that many people in the groups personally concealed facts about their own travel history, information regarding their health conditions, upper middle class segregation theories that they remain in safe space created the false impression of the health risk. Many people though agreed that the virus was imported from abroad and that lowest income group people with no travel history were not the ones risking others and yet they exchanged 
information calling out the vulnerable, identifying people based on fake and over-reacted news ultimately leading to several instances of prejudice, hate and large scale violence and disruptions. A detailed debunking message correlated positively with the debunking effect. Surprisingly, however, a detailed debunking message also correlated positively with the misinformation-persistence effect (Sally et al 2017) therefore the process of debunking must be followed with a replacement of the fake with correct and verified information (Nyhan \& Reifler 2015). Changing ways news information is being accessed, new attitudes about what it means to be informed, and a youth preference for opinionated rather than objective news. This does not indicate that young people disregard the basic ideals of professional journalism but, rather, that they desire more authentic renderings of them (Marchi 2012). Perceived realism of fake news is stronger among individuals with high exposure to fake news and low exposure to hard news than among those with high exposure to both fake and hard news (Balmas 2014). To combat false information people need to learn to spot patterns in misinformation. For instance, breaking news events usually result in a spate of unverified information on social media and it is now a pattern in which major incidents are followed by widespread misinformation. Doctored videos, edited posts, and unverified data come as a spurt of information even as reporters scramble to verify facts in times of exigency works as a pattern. This pattern compels us to slow down and follow a story over time and verify through a variety of sources eye witnesses or thought leaders before sharing and acting on initial reports.

This is a part of the ongoing research and will be updated as we analyze more information. Acknowledgement

The first author (Syeda Saadia Azim) would like to acknowledge Sister Nivedita University for support and like to thank Prof. Buroshiva Dasgupta, noted Journalist and Academic, for his constant guidance. Co-author, Arindam Roy would like to thank the South Asian Forum for Environment (SAFE) for overall support.

\section{References}

Allcott, H., \& Gentzkow, M. (2017). Social media and fake news in the 2016 election. Journal of economic perspectives, 31(2), 211-36.

Balmas, M. (2014). When fake news becomes real: Combined exposure to multiple news sources and political attitudes of inefficacy, alienation, and cynicism. Communication research, 41(3), 430-454.

Berinsky, A. J. (2017). Rumors and health care reform: Experiments in political misinformation. British journal of political science, 47(2), 241-262.. 
Brewer, P. R., Young, D. G., \& Morreale, M. (2013). The impact of real news about "fake news": Intertextual processes and political satire. International Journal of Public Opinion Research, 25(3), 323-343.

Chan, M. P. S., Jones, C. R., Hall Jamieson, K., \& Albarracín, D. (2017). Debunking: A meta-analysis of the psychological efficacy of messages countering misinformation. Psychological science, 28(11), 1531-1546.

Gupta, A., Lamba, H., Kumaraguru, P., \& Joshi, A. (2013, May). Faking sandy: characterizing and identifying fake images on twitter during hurricane sandy. In Proceedings of the 22nd international conference on World Wide Web (pp. 729-736).

Lazer, D. M., Baum, M. A., Benkler, Y., Berinsky, A. J., Greenhill, K. M., Menczer, F., \& Metzger, M. J. \& Zittrain, JL (2018). The science of fake news. Science, 359(6380), 1094-1096.

Marchi, R. (2012). With Facebook, blogs, and fake news, teens reject journalistic "objectivity". Journal of communication inquiry, 36(3), 246-262.

Nyhan, B., \& Reifler, J. (2015). Displacing misinformation about events: An experimental test of causal corrections. Journal of Experimental Political Science, 2015. doi: 10.1017/XPS.2014.22.

Pennycook, G., \& David, G. (2018). Rand. 2018. Who falls for fake news? The roles of bullshit receptivity, overclaiming, familiarity, and analytic thinking. Journal of Personality, 63.

Rubin, V. L. (2010). On deception and deception detection: Content analysis of computer-mediated stated beliefs. Proceedings of the American Society for Information Science and Technology, 47(1), 1-10.

Zubiaga, A., Liakata, M., Procter, R., Wong Sak Hoi, G., \& Tolmie, P. (2016). Analysing how people orient to and spread rumours in social media by looking at conversational threads. PloS one, 11(3), e0150989.

Berduygina, O. N., Vladimirova, T. N., \& Chernyaeva, E. V. (2019). Trends in the spread of fake news in mass media. Media Watch, 10(1), 122-132.

Bharali, B., \& Goswami, A. L. (2017). Fake news: Credibility, cultivation syndrome and the new age media. Media Watch, 9(1), 118-130.

Bali, A., \& Desai, P. (2019). Fake News and Social Media: Indian Perspective. Media Watch, 10(3), 737-750.

Azim, S. S., Roy, A., Aich, A., \& Dey, D. (2020). Fake news in the time of environmental disaster: Preparing framework for COVID-19. 


\section{Supplementary information}

\section{Details of different fake news and its origin}

1. Use of Hydroxy chloroquine - the anti malarial drug: In the first and second week of April 2020, Hydroxy Choloroquine was the top medicine prescribed on WhatsApp in India. There wasn't ever a proper doctor's prescription or even directives to use it till then.

(https://www.boomlive.in/fast-check/false-pak-citizens-demand-hydroxychloroquine-inst ead-of-kashmir-7713).

2. Garlic and hot water were effective protectors: It took more than two weeks to debunk the misinformation through several debunking outlets such as Altnews or Indian Scientists Response to COVID-19. But before that people treated each other on the increased dependence on garlic in hot water.

3. Cow Urine can cure COVID-19: The message did the rounds as hot cakes on WhatsApp for several weeks in several of the social media groups before mainstream media debunked the story some later. (https://timesofindia.indiatimes.com/life-style/health-fitness/health-news/coronavirus-be ware-of-these-dangerous-myths-about-the-wuhan-virus/photostory/73937059.cms)

4. Pneumonia vaccines/antibiotics help mitigate COVID- was the message about vaccines and how those against pneumonia and other antibiotics protected people against the new coronavirus.

5. Indians have stronger immunity- mainstream newspaper Livemint opinion claimed that COVID-19 will not affect Indians as they have the genetics for a sturdier immune system. https://www.livemint.com/elections/opinion/it-makes-no-sense-for-us-to-panic-over-the-c oronavirus-11583679515225.html

6. BCG and other vaccines: The claim that the anti-tuberculosis Bacillus Calmette-Guérin (BCG) vaccine protected people against corona virus infection was discredited by the World Health Organization that had to clarify that there was no evidence to suggest that BCG vaccine could protect people against Covid-19.

7. Warm Climate and Young Population: A survey by mainstream media disclosed that $65 \%$ Indians thought Corona Virus did not spread in warm climates. https://www.news18.com/news/buzz/65-indians-think-covid-19-wont-affect-india-as-its-a -warm-country-survey-reveals-2544649.html

8. Non Vegetarian Food: Most cities in West India had shut down poultry and fish shops and did not count them as essential food products during the initial phases of lockdown 
that began on March 24, 2020. More than fifty percent of the respondents believed that the

9. Preventive measures through Yoga, Ayurvedic and Homeopathic medicines such as Arsenicum album 30c and files of Camphor could prevent the deadly virus. While several medicine shops sold the India's AYUSH Ministry-the ministry of indigenous alternative medicine promoted several preventive medicines without any basis.

10. Mainstream newspaper the "Times of India" published a report on May 22, with the headline that read, "Not just sins Ganga water can end Coronavirus" quoting an Eminent river engineer and former professor of civil engineering at IIT in the Banaras Hindu University (BHU) said that the judicious use of river technology can help resolve the Coronavirus crisis as well as the plight of Ganga river. 